\title{
Characteristics of Leiomyosarcoma: Induction of Hematogenous Metastasis by Isolated Uterine Mesenchymal Tumor Stem-like Cells
}

\author{
TAKUMA HAYASHI ${ }^{1}$, KENJI SANO ${ }^{2}$, TOMOYUKI ICHIMURA ${ }^{3}$, YAE KANAI $^{4}$, DORIT ZHARHARY ${ }^{5}$, \\ HIROYUKI ABURATANI ${ }^{6}$, NOBUO YAEGASHI ${ }^{7}$ and IKUO KONISHI ${ }^{8}$ \\ ${ }^{1}$ Department of Molecular and Cellular Immunology, \\ Shinshu University Graduate School of Medicine, Nagano, Japan; \\ ${ }^{2}$ Department of Medical Laboratory, Shinshu University Hospital, Nagano, Japan; \\ ${ }^{3}$ Department of Obstetrics and Gynecology, Osaka City University Graduate School of Medicine, Osaka, Japan; \\ ${ }^{4}$ Department of Pathology, Keio University School of Medicine, Tokyo, Japan; \\ ${ }^{5}$ SIGMA-Aldrich Israel, Rehovot, Israel; \\ ${ }^{6}$ RCAST, The University of Tokyo, Tokyo, Japan; \\ ${ }^{7}$ Department of Obstetrics and Gynecology, Tohoku University School of Medicine, Miyagi, Japan; \\ ${ }^{8}$ National Hospital Organization Kyoto Medical Center, Kyoto, Japan
}

\begin{abstract}
Background/Aim: Uterine leiomyosarcoma (Ut$L M S)$ is a refractory tumor that repeatedly recurs with hematogenous metastasis, which may be due to the presence of drug-resistant tumor stem cells. Its treatment is limited to surgical procedures. We previously reported that Ut-LMS spontaneously developed in mice deficient in the proteasome component low-molecular mass polypeptide 2 (LMP2). We showed that LMP2 expression was significantly attenuated specifically in human Ut-LMS. The aim of this study was to investigate the role of LMP2 in hematogenous metastasis using xenograft models with tumor stem-like cells. Materials and Methods: We isolated tumor stem-like cells from LMP2negative primary human Ut-LMS cells established from a human Ut-LMS tissue using the side population (SP) procedure. These cells were used to develop xenograft models with tumor stem-like cells. Results: Human Ut-LMS stem-like cells showed stronger hematogenous metastatic potential than normal Ut-LMS cells. Tumor stem-like cells also had the potential to differentiate into vascular endothelial cells
\end{abstract}

This article is freely accessible online.

Correspondence to: Takuma Hayashi D.M.Sci, M.S., Section Head of Cancer Medicine, National Hospital Organization Kyoto Medical Center, Fushimi-ku, Kyoto, Japan. Tel: +81 0263372615, e-mail: yoyoyo224@hotmail.com

Key Words: Leiomyosarcoma, tumor stem cell, angiogenesis, hematogenous metastasis. through VEGF-A signaling. Conclusion: These results reflect frequent hematogenous metastasis by human Ut-LMS in clinical settings, and may lead to the development of treatments that inhibit hematogenous metastasis in Ut-LMS.

Uterine leiomyosarcoma (Ut-LMS) is an intractable gynecological malignancy that repeatedly recurs and metastasizes, and its pathogenesis has not yet been elucidated in detail $(1,2)$. According to clinical findings, Ut-LMS cooccurs with uterine leiomyoma (LMA), and mostly appears together with leiomyoma. The best treatment for Ut-LMS and uterine LMA is surgery $(3,4)$. Due to current social standards, which require preservation of the uterus, effective treatments need to be established for these uterine mesenchymal tumors. Malignant tumor stem cells are more resistant to antitumor agents and radiation than normal malignant tumor cells. Therefore, a major cause of the recurrence of malignant tumors after clinical treatments with antitumor agents and/or radiation is the presence of malignant tumor stem cells.

In collaboration with Professor Susumu Tonegawa (Picower Institution at M.I.T.), we have previously reported that spontaneous Ut-LMS in low-molecular mass polypeptide 2 (LMP2)-deficient mice had hematogenous metastatic potential (5-8) (Figure 1B). We have also demonstrated that the majority of human Ut-LMS tissues exhibited the weak or negative expression of LMP2 (9-10). Therefore, we isolated human Ut-LMS stem-like cells from LMP2-negative SK-LMP-1 primary cultured cells established from human Ut-LMS tissue using the standard side population (SP) procedure and have been examining 
A Vascular endothelial growth factor signaling

B

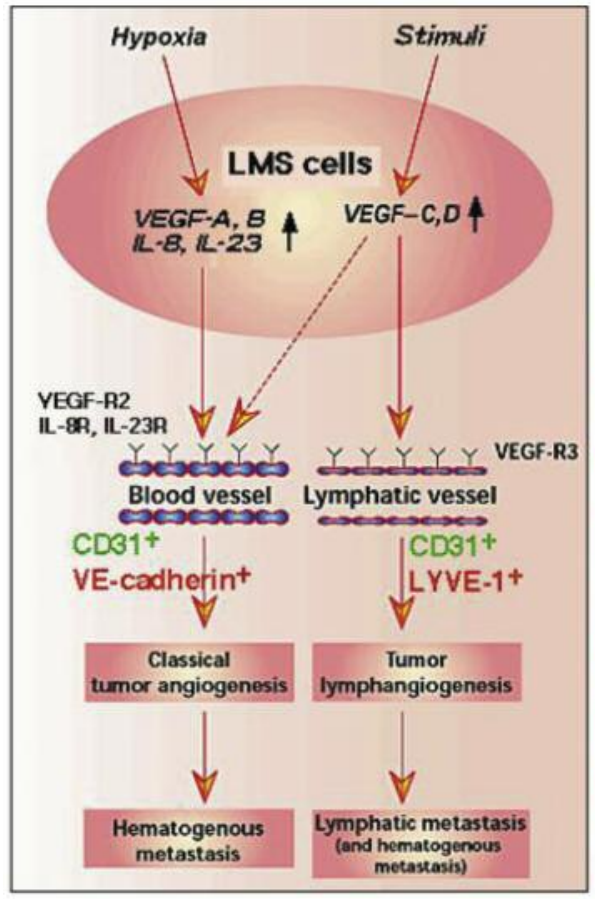

Uterine LMS hematogenous
metastasis in Lmp2- mice

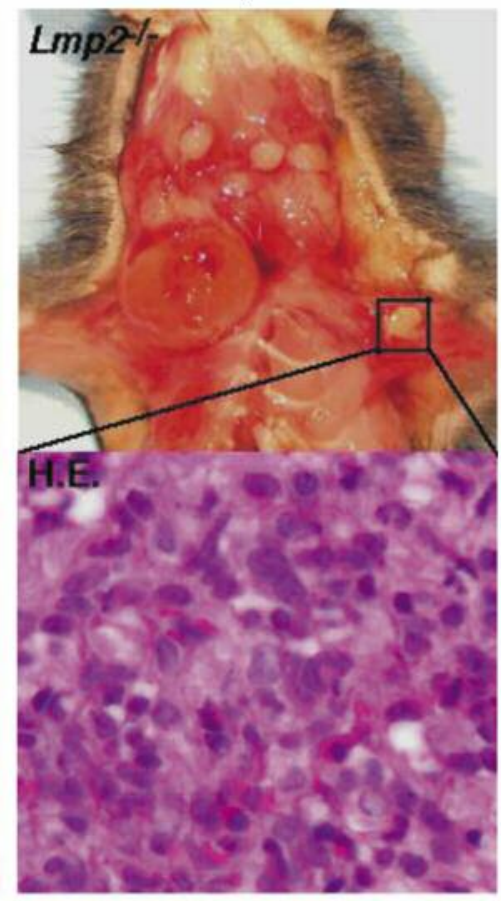

Figure 1. Angiogenesis and lymphangiogenesis signals. A. Dissecting tumor angiogenesis. Hypoxic tumor cells produce VEGF-A or VEGF-B, which binds to and activates VEGFR-2 on CD31 and CD144 (VE-CADHERIN) double-positive vascular endothelial cells, leading to classical tumor angiogenesis. Tumors that secrete VEGF-C or VEGF-D may also induce lymphangiogenesis by activating VEGFR-3 on CD31 and LYVE-1 doublepositive lymphatic endothelial cells of lymphatic vessels, a process known as tumor lymphangiogenesis. Classical tumor angiogenesis has been shown to correlate with hematogenous metastasis. In animal models, the induction of lymphangiogenesis by VEGF-C or VEGF-D led to an increase in tumor metastasis via the lymphatic system. B. Spontaneous uterine leiomyosarcoma (Ut-LMS) in low-molecular mass polypeptide 2 (LMP2)deficient mice exhibited hematogenous metastatic potential.

their physiological characteristics. The purpose of the present study was to apply our findings to the development of a new clinical treatment for uterine mesenchymal tumors.

\section{Materials and Methods}

Antibodies and cell line. We purchased the established human LMS primary cell line, SK-LMS (ATCC ${ }^{\circledR}$ HTB-88 ${ }^{\mathrm{TM}}$ ) from the American Type Culture Collection (ATCC) (Manassas, VA, USA). Antibodies for CD31 (bs-0195R), CD34 (bs-2038R), CD144 (VE-CADHERIN) (bs-4310R), and LYVE-1 (bs-1311R) were purchased from Bioss Inc. and used in the FACS analysis and molecular pathological studies (Woburn, MA, USA). Alexa Fluor ${ }^{\circledR} 488$ conjugated goat anti-mouse $\operatorname{IgG}(\mathrm{H}+\mathrm{L})$ and Alexa Fluor ${ }^{\circledR} 546$ conjugated goat antirabbit $\operatorname{IgG}(\mathrm{H}+\mathrm{L})$ secondary antibodies were purchased from Invitrogen (Waltham, MA, USA). DAPI Mounting Medium was purchased from Vector Laboratories, Inc. (Burlingame, CA, USA).

Plasmid DNA transfection and BD FACSAria ${ }^{T M}$ III analysis. We produced a $P L M P 2$-pro-EGFP vector that mediates expression of the enhanced green fluorescence protein (EGFP) via the $L M P 2$ promoter using the $p E G F P$ vector (Clontech Laboratories, Inc., Mountain View, CA, USA). SK-LMS-1 cells were transfected with the pLMP2-proEGFP vector in complete medium using FuGENE ${ }^{\circledR}$ HD (Promega, Madison, WI, USA) according to the manufacturer's protocol. Transfected SK-LMS-1 cells were cultured in complete medium containing G-418 (final concentration of $100 \mu \mathrm{g} / \mathrm{ml}$, SIGMA-Aldrich, Tokyo, Japan) at $37^{\circ} \mathrm{C}$ for $48 \mathrm{~h}$. After $48 \mathrm{~h}$ in culture, pEGFP-SKLMS- 1 cells were collected by centrifugation at $900 \times g$ at $4^{\circ} \mathrm{C}$ for 5 min. Cells not expressing LMP2 were selected as EGFP-negative cells (Figure 2). To distinguish the two cell populations, EGFPpositive and -negative cells, gating was performed on EGFP-negative cells and sorting was conducted using BD FACSAria ${ }^{\mathrm{TM}}$ III (Becton, Dickinson and Company, Franklin Lakes, NJ, USA) at 1,000 events/second in the high purity mode (Figure 2). The sensitivity of the detector was adjusted and gated using pEGFP-SK-LMS-1 cells. The purity of EGFP-negative SK-LMS-1 cells, namely, LMP2 nonexpressing cells, was $98.8 \%$ (Figure 2).

Preparation of cells by the SP method and sorting on BD FACSAria ${ }^{T M}$ III. The SP method using specific markers (CD133 and CD34) for tumor stem cells and the dye selectivity of the $\mathrm{ABC}$ transporter are widely used to isolate tumor stem cells. Approximately 106 SK-LMS- 
Isolation of LMP2-negative KS-LMS-1 cells

Human Chr. $601 \mathrm{MDD}$

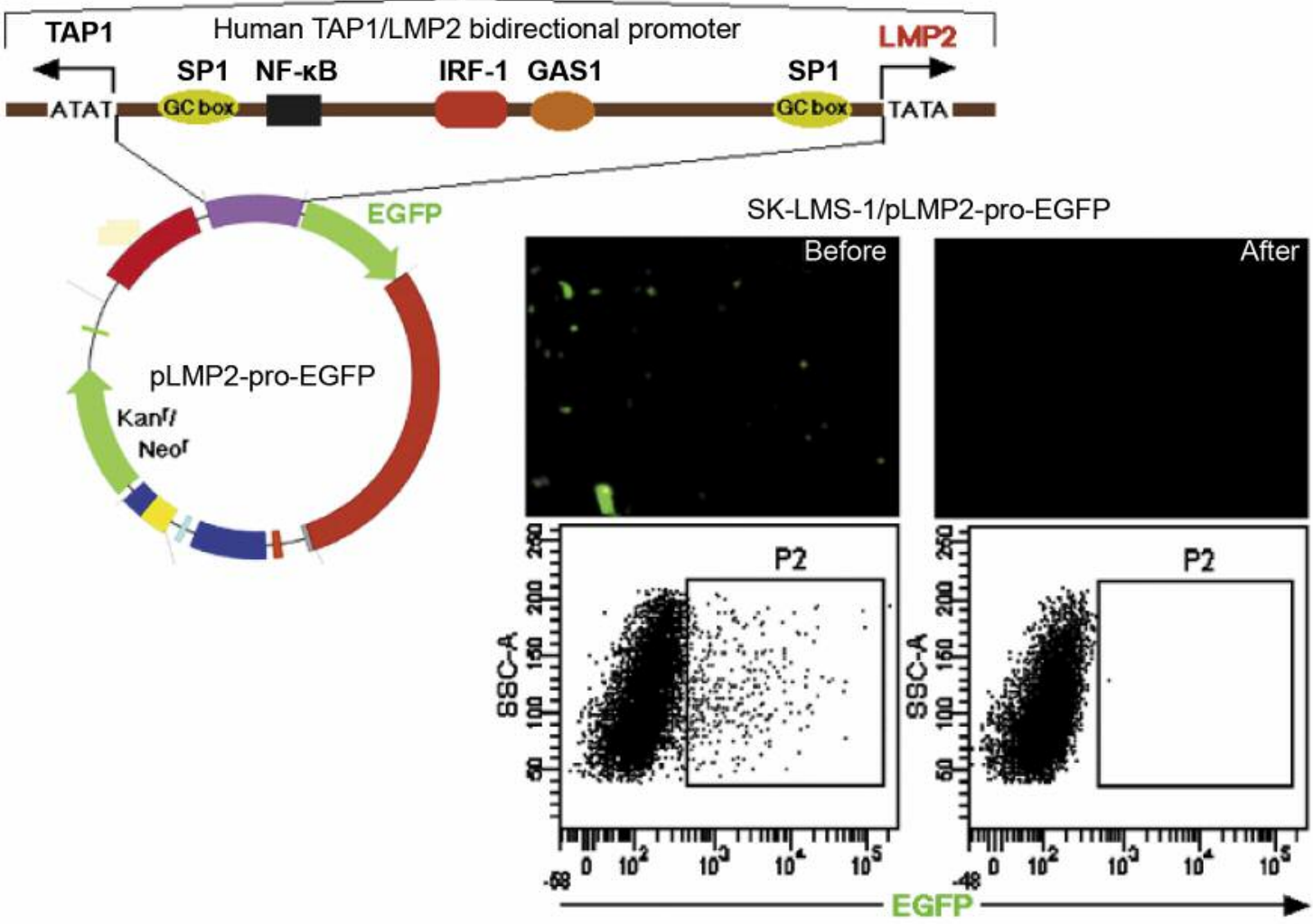

Figure 2. Isolation of LMP2-negative SK-LMP-1 cells by BD FACSAria. We produced pLMP2-pro-EGFP, which mediated the expression of the enhanced green fluorescence protein (EGFP) as a fusion with LMP2 via the LMP2 enhancer/promoter, based on the pEGFP vector to purify LMP2 non-expressing and LMP2-expressing SK-LMP-1 cells by BD FACSAria.

1/LMP2(-) cells/ml were resuspended in warmed staining medium and 10-ml aliquots were transferred into 50-ml tubes. Fifty-microliter aliquots of Hoechst 33342 (Bis-Benzimide, SIGMA-Aldrich, Tokyo, Japan) stock solution $(1 \mathrm{mg} / \mathrm{ml})$ were added to each tube (final concentration of $5 \mu \mathrm{g} / \mathrm{ml}$ ) and incubated in a water bath at $37^{\circ} \mathrm{C}$ for $90 \mathrm{~min}$. Forty milliliters of cold wash buffer were added at once to each 50-ml tube to stop the reaction. Suspended cells were centrifuged at $900 \times g$ for $5 \mathrm{~min}$. In order to further stain cells with the fluorescent antibody for CD34, the cell pellet was resuspended in washing buffer to $106 / 100 \mu \mathrm{l}$, and $1 \mu \mathrm{g}$ of the anti-CD34 antibody was added. After the cell suspension had been incubated on ice for $30 \mathrm{~min}, 20$ volumes of wash buffer were added to the cell suspension, which was then subjected to centrifugation at $900 \times g$ at $4^{\circ} \mathrm{C}$ for $5 \mathrm{~min}$. Secondary antibody staining was also performed in the same manner. The cell pellet was resuspended to approximately $5 \times 10^{6}$ cells $/ \mathrm{ml}$ in cold PBS to which propidium iodide (PI) (SIGMA-Aldrich, Tokyo, Japan) had been added and the cell suspension was then passed through a cell strainer (Corning, New York, NY, USA). Then, FACS was set up to isolate the SP cell fraction and the major population (MP) fraction located above the SP cell fraction. We distinguished human Ut-LMS stem-like cells (SP/LMP2(-)) and normal Ut-LMS cells (MP/LMP2(-
)) from SK-LMS-1/LMP2(-) using the SP method as the established procedure for the isolation of cancer stem cells, and we then cultured both isolated cells in STEMPRO ${ }^{\circledR}$ MSC SFM (Invitrogen, Waltham, MA, USA).

Xenograft studies for the micrometastasis model. SP/LMP2(-), MP/LMP2(-), or SK-LMP-1 cells were harvested from a culture using trypsin, washed, and resuspended in PBS $\left(2 \times 10^{7}\right.$ cells $\left./ \mathrm{ml}\right)$. Nude mice (BALB/cSlc-nu/nu, female, 7-8 weeks old, Japan SLC, Shizuoka, Japan) were injected with $1 \times 10^{6}$ SP/LMP2(-), MP/LMP2(-), or SK-LMP-1 cells in $5 \mathrm{mg} / \mathrm{ml} \mathrm{BD} \mathrm{Matrigel} \mathrm{Matrix}$ (BD Biosciences, San Jose, CA, USA) in culture medium containing $15 \%$ fetal calf serum plus SmGM-2 SingleQuots (Lonza, Basel, Switzerland) at a volume of $100 \mu$ on the left side of the second mammary fat pad. Tumor formation was assessed every day. Tumor volumes were calculated as $(\mathrm{L} \times \mathrm{W} \times \mathrm{W}) / 2$, where $\mathrm{W}$ represents width and $L$ represents length. Statistical analyses were performed on mean tumor volumes at the end of the study using the Student's $t$-test.

Xenografted BALB/c nu/nu mice were sacrificed for molecular pathological studies 2 months after the injection. Whole lung and primary tumor tissues were also harvested for hematoxylin and 

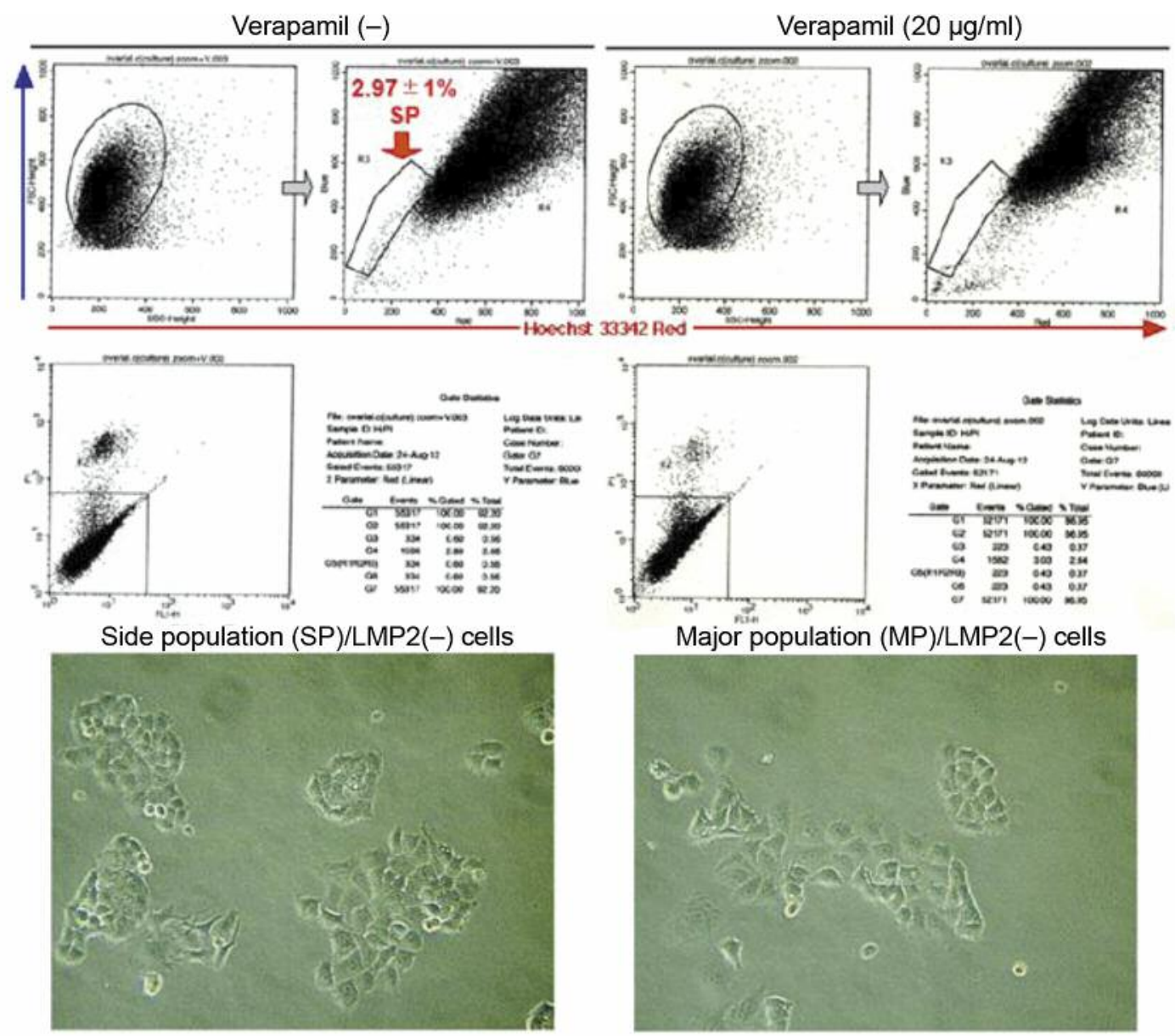

Figure 3. Preparation of side population (SP) cells by sorting with BD FACSAria. The SP method using specific markers (CD133, CD34, and CD44) for tumor stem cells and the dye selectivity of $A B C$ transporters is widely used as a method to isolate tumor stem cells. Approximately $10^{6}$ cells $/ m l$ SK-LMS-1/LMP2(-) cells were resuspended in warmed staining medium and 10-ml aliquots were transferred into 50-ml tubes. Fifty-microliter aliquots of Hoechst 33342 (Bis-Benzimide, SIGMA-Aldrich) stock solution $(1 \mathrm{mg} / \mathrm{ml}$ ) were added to each tube (final concentration of $5 \mu \mathrm{g} / \mathrm{ml}$ ), which were then incubated in a water bath at $37^{\circ} \mathrm{C}$ for $90 \mathrm{~min}$. Regarding fluorescence staining, the cell pellet was suspended in washing buffer to $10^{6 / 100} \mu l$, and $1 \mu \mathrm{g}$ of the anti-CD34 antibody was added to the cell suspension, which was subjected to $900 \times \mathrm{g}$ at $4^{\circ} \mathrm{C}$ for $5 \mathrm{~min}$. The cell pellet was resuspended to approximately $5 \times 10^{6}$ cells $/ \mathrm{ml}$ in cold PBS to which propidium iodide (PI) (SIGMA-Aldrich) had been added and was then passed through the Cell Strainer (Corning). FACS was set up to isolate cancer stem-like cells as follows and the side population (SP) cell fraction and the major population (MP) fraction were sorted. SK-LMS-1/LMP2(-) cells gated on SP cell fractions were sorted as SP cells and SK-LMS-1/LMP2(-) gated on the outside of the SP cell fraction were sorted as MP cells. We distinguished human Ut-LMS stem-like cells [SP/LMP2(-)] and normal UtLMS cells [MP/LMP2(-)] from SK-LMS-1/LMP2(-) using the SP method as the established procedure for the isolation of cancer stem cells, and then cultured both isolated cells in STEMPRO ${ }^{\circledR}$ MSC SFM (Invitrogen). By adding $20 \mu \mathrm{g} / \mathrm{ml}$ reserpine to the SP/LMP2(-) cell suspension, it was confirmed that the ABCG2 transporter on the cell membrane of SP was inhibited and the SP fraction disappeared (upper right chart of FACS).

eosin (H\&E) staining. Harvested tumors in primary sites and whole lungs after the indicated times were fixed and embedded in paraffin, sectioned, and subjected to H\&E staining following the standard procedure. The number of SP/LMP2(-), MP/LMP2(-), or SK-LMP1 tumor nodules in the alveolar tissues of mice was counted 2 months after the injection under a microscope (Nikon COOLSCOPE, Tokyo, Japan). To calculate the concentrations of VEGF-A, VEGF-B, VEGF-C, and VEGF-D (Figure 1A), samples of tumor cellular extracts collected from xenografted BALB/c nu/nu were subjected to ELISA using OptEIA ${ }^{\mathrm{TM}}$ Set, VEGF-A, VEGF-B, VEGF-C, and VEGF-D (BD Biosciences, San Jose, CA, USA).
Immunohistochemistry (IHC). IHC staining for CD31, CD144 (VECADHERIN), and LYVE-1 was performed on tissue sections of xenografts derived from BALB/c $n u / n u$-injected with SP/LMP2(-), MP/LMP2(-), or SK-LMS-1 cells. Briefly, tumor tissue sections were incubated with the appropriate primary antibodies at $4{ }^{\circ} \mathrm{C}$ overnight. We used rabbit polyclonal antibodies to CD144 (VECADHERIN) (1:200) or LYVE-1 (1:200), a mouse monoclonal antibody to CD31 (1:200), as primary antibodies. Following an incubation with the secondary antibody, i.e., the Alexa Fluor ${ }^{\circledR} 488$ conjugated anti-mouse IgG antibody or Alexa Fluor ${ }^{\circledR}$ 546-conjugated anti-rabbit IgG antibody (1:200; Invitrogen), sections were washed 
A

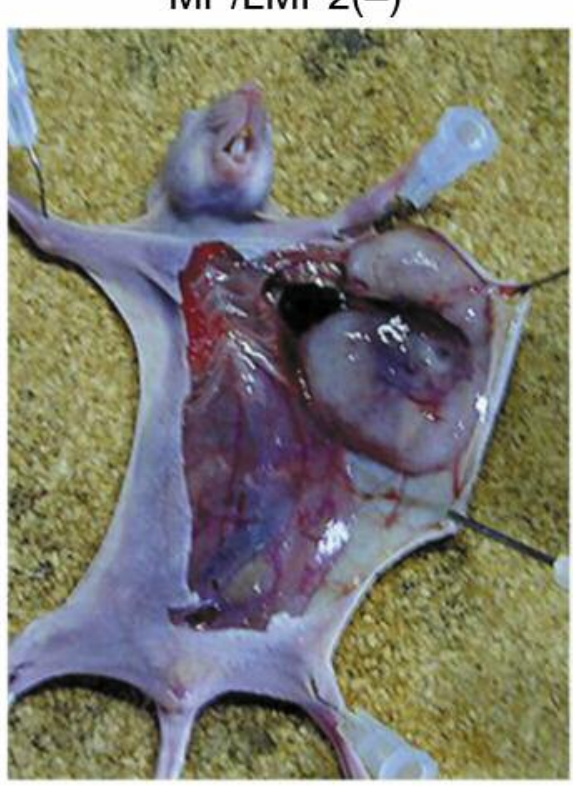

SP/LMP2(-)

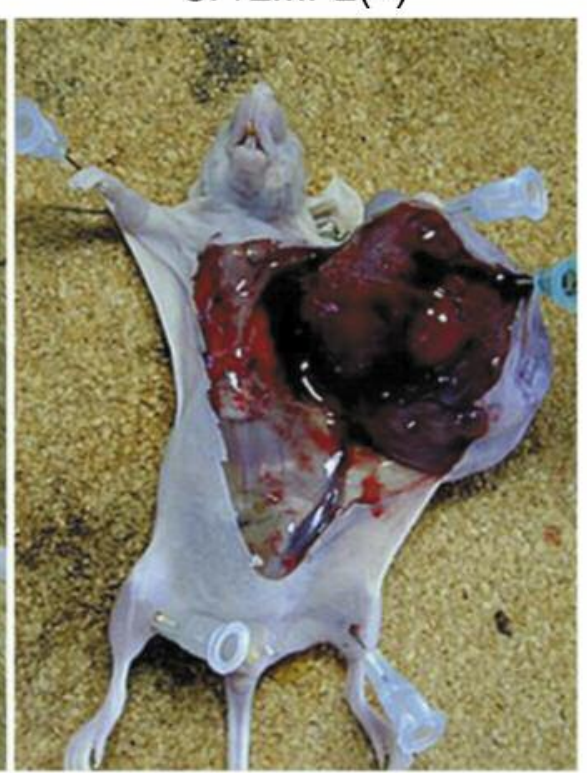

B

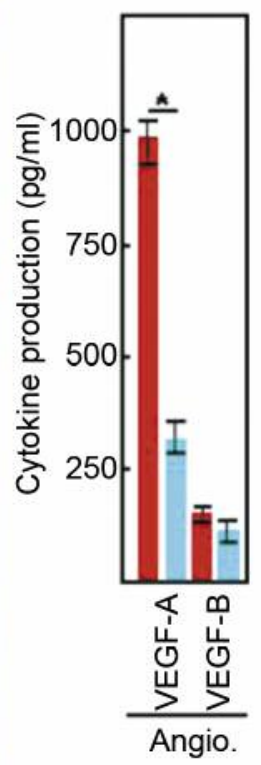

C

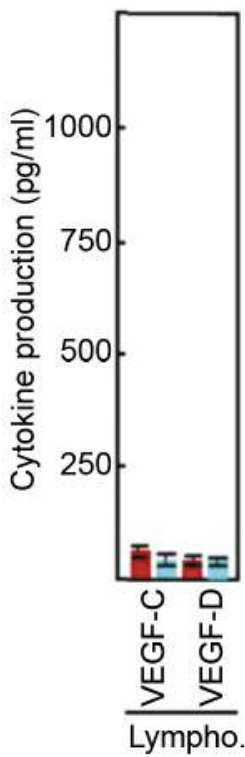

Figure 4. Enhanced VEGF-A secretion from xenografts derived from human Ut-LMS stem-like cells. A. Photographs show xenografts derived from $B A L B / c$ nu/nu mice inoculated with MP/LMP2(-) or SP/LMP2(-) 2 months after the injection. Xenografts derived from BALB/c nu/nu mice inoculated with $S P / L M P 2(-)$ were a highly angiogenetic malignancy. Data are representative of one out of ten independent experiments obtained with different xenograft samples derived from SP/LMP2(-) or MS/LMP2(-) cells. B and C. Various growth factors (B. angiogenesis factors; VEGF$A$ and VEGF-B, C. Lymphangiogenesis factors; VEGF-C and VEGF-D) produced by vascular endothelial cells and lymphatic endothelial cells in xenografts derived from SP/LMP2(-) and MP/LMP2(-) were measured by the OptEIA ${ }^{T M}$ set. The results obtained revealed that the secreted levels of vascular endothelial growth factor (VEGF), particularly VEGF-A, were significantly higher in SP/LMP2(-) than in MP/LMP2(-) xenografts. ${ }^{*} p<0.001$. In xenograft experiments using nude mice, similar tumorigenicity was observed for SP/LMP2(-) and MP/LMP2(-) xenografts.

and coverslipped with mounting medium and 40,6-diamidino-2phenylindole (DAPI) (Vectashield; Vector Laboratories, Burlingame, CA, USA) and then visualized under a confocal microscope (Leica TCS SP8, Wetzlar, Germany) according to the manufacturer's procedure. Normal rabbit or mouse antiserum was used as a negative control for the primary antibody. These experiments with BALB/c $n u / n u$ mice were conducted at Shinshu University in accordance with institutional guidelines (approval no. M192).

\section{Results}

We produced the $p L M P 2-p r o-E G F P$ vector, which encodes EGFP as a fusion with LMP2. SK-LMS-1 cells were transfected with the $p L M P 2-p r o-E G F P$ vector in complete medium using FuGENE ${ }^{\circledR}$ HD with the standard transfection procedure, and the expression of EGFP was confirmed after $48 \mathrm{~h}$ using a fluorescence microscope (Figure 2). We isolated a candidate population of human Ut-LMS stem-like cells from human Ut-LMS primary cells, SK-LMS-1 cells, using the SP method $(11,12)$. Previous studies have reported that SP cells exhibit cancer stem cell activity in various organs and tissues (13-15). We analyzed the presence of an average of $2.97 \pm 1 \%$ human Ut-LMS SP cells (SP/LMP2(-) cells) among SK-LMS-1 cells (Figure 3). By adding $50 \mu \mathrm{M}$ reserpine to the SP/LMP2(-) cell suspension, we confirmed that the ATP-binding cassette super-family $G$ member 2 (ABCG2) transporter on the cell membrane of SP was inhibited and the SP fraction disappeared (Figure 3).

Tumor growth was clearly observed in control BALB/c $n u / n u$ mice inoculated with the MP/LMP2(-) cell (normal human LMS cell) fraction; however, a reduction in tumor growth was not observed in BALB/c nu/nu mice inoculated with SP/LMP2(-) cells (human LMS stem-like cells) (Figure 4A, Table I). No significant differences were observed in xenograft growth between MP/LMP2(-) and SP/LMP2(-) cells (Figure 5).

Xenografts derived from BALB/c nu/nu mice inoculated with SP/LMP2(-) cells were a higher angiogenic malignancy than those derived from BALB/c nu/nu mice inoculated with MP/LMP2(-) cells (Figure 4A). Therefore, various vascular endothelial growth factors (VEGFs) (Figure 1A), namely, VEGF-A, VEGF-B, VEGF-C, and VEGF-D derived from the primary tumors of $\mathrm{BALB} / \mathrm{c} n u / n u$ mice xenografted with SP/LMP2(-) or MP/LMP2(-) cells were measured using the $\mathrm{OptEIA}^{\mathrm{TM}}$ set. The expression levels of VEGFs, particularly 
Table I. Characteristics of xenografts derived from MP/LMP2(-) or SP/LMP2(-).

\begin{tabular}{|c|c|c|c|c|c|c|c|c|c|c|c|c|}
\hline \multirow[t]{3}{*}{ Clone\# } & \multirow{3}{*}{$\begin{array}{l}\text { Volume } \\
\left(\mathrm{mm}^{3}\right)\end{array}$} & \multirow[t]{3}{*}{ M } & \multicolumn{2}{|c|}{ Vascular } & \multicolumn{2}{|c|}{ Lymphatic } & \multicolumn{3}{|c|}{ Core (tumor) } & \multicolumn{3}{|c|}{ Capsule } \\
\hline & & & \multicolumn{2}{|c|}{$\mathrm{pg} / \mathrm{ml}$ in xenografts } & \multicolumn{2}{|c|}{$\mathrm{pg} / \mathrm{ml}$ in xenografts } & \multicolumn{3}{|c|}{ Expression level on IHC } & \multicolumn{3}{|c|}{ Expression level on IHC } \\
\hline & & & VEGF-A & VEGF-B & VEGF-C & VEGF-D & CD31 & CD144 & LYVE-1 & CD31 & $\mathrm{CD} 144$ & LYVE-1 \\
\hline MP/LMP2(-)1 & 387 & - & 328 & 121 & 54 & 48 & $+/-$ & $+/-$ & - & - & +++ & - \\
\hline MP/LMP2(-)2 & 382 & - & 289 & 127 & 53 & 37 & $+/-$ & $+/-$ & - & - & +++ & - \\
\hline MP/LMP2(-)3 & 363 & - & 261 & 111 & 41 & 30 & - & - & - & - & +++ & - \\
\hline MP/LMP2(-)4 & 460 & + & 346 & 138 & 62 & 56 & + & + & - & - & ++ & - \\
\hline MP/LMP2(-)5 & 433 & - & 312 & 119 & 50 & 51 & $+1-$ & $+/-$ & - & - & +++ & - \\
\hline MP/LMP2(-)6 & 373 & - & 273 & 109 & 42 & 34 & - & - & - & - & +++ & - \\
\hline MP/LMP2(-)7 & 412 & + & 299 & 135 & 45 & 52 & $+/-$ & $+/-$ & - & - & +++ & - \\
\hline MP/LMP2(-)8 & 392 & - & 301 & 130 & 49 & 45 & $+/-$ & $+/-$ & - & - & +++ & - \\
\hline MP/LMP2(-)9 & 403 & + & 332 & 118 & 58 & 44 & + & $+/-$ & - & - & +++ & - \\
\hline MP/LMP2(-)10 & 380 & - & 295 & 116 & 47 & 41 & - & $+/-$ & - & - & ++ & - \\
\hline SP/LMP2(-)1 & 442 & + & 942 & 145 & 54 & 45 & +++ & +++ & - & - & +++ & - \\
\hline SP/LMP2(-)2 & 401 & + & 883 & 132 & 55 & 32 & ++ & +++ & - & - & +++ & - \\
\hline SP/LMP2(-)3 & 394 & + & 869 & 130 & 60 & 43 & ++ & +++ & - & - & +++ & - \\
\hline SP/LMP2(-)4 & 471 & + & 1013 & 148 & 72 & 55 & +++ & +++ & - & - & +++ & - \\
\hline SP/LMP2(-)5 & 375 & + & 815 & 124 & 51 & 35 & ++ & ++ & - & - & +++ & - \\
\hline SP/LMP2(-)6 & 389 & - & 843 & 128 & 58 & 37 & ++ & ++ & - & - & ++ & - \\
\hline SP/LMP2(-)7 & 382 & + & 905 & 133 & 54 & 53 & +++ & ++ & - & - & +++ & - \\
\hline SP/LMP2(-)8 & 430 & + & 982 & 140 & 70 & 40 & +++ & +++ & - & - & +++ & - \\
\hline SP/LMP2(-)9 & 403 & + & 965 & 138 & 67 & 50 & +++ & +++ & - & - & +++ & - \\
\hline SP/LMP2(-)10 & 412 & + & 921 & 134 & 62 & 47 & +++ & +++ & - & - & +++ & - \\
\hline SK-LMP-1\#1 & 327 & - & 302 & 98 & 37 & 25 & $+/-$ & + & - & - & +++ & - \\
\hline SK-LMP-1\#2 & 341 & - & 315 & 87 & 32 & 29 & $+/-$ & + & - & - & +++ & - \\
\hline
\end{tabular}

Volume $\left(\mathrm{mm}^{3}\right)$ : Volumes of xenografts were calculated as $(\mathrm{L} \times \mathrm{W} \times \mathrm{W}) / 2$, where $\mathrm{W}$ represents width and $\mathrm{L}$ represents length. M: the numbers of micrometastases derived from SP/LMP2(-), MP/LMP2(-), or SK-LMP-1 counted as tumor nodules in the alveolar tissues of mice after 2 months of injections under the microscope. To calculate the concentrations of several cytokines, ELISA in tumor extracts collected from xenografted BALB/c $n u / n u$ were performed using OptEIA ${ }^{\mathrm{TM}}$ Set, VEGF-A, VEGF-B, VEGF-C, and VEGF-D. Expression levels of specific cell surface markers, CD31, CD144 (VE-CADHERIN), and LYVE-1 for vascular or lymphatic endothelial cells examined by IHC, and observed under the microscope, -: Negative, no stained cells; +/-: partial expression; +: low expression; ++: medium expression; +++: high expression.

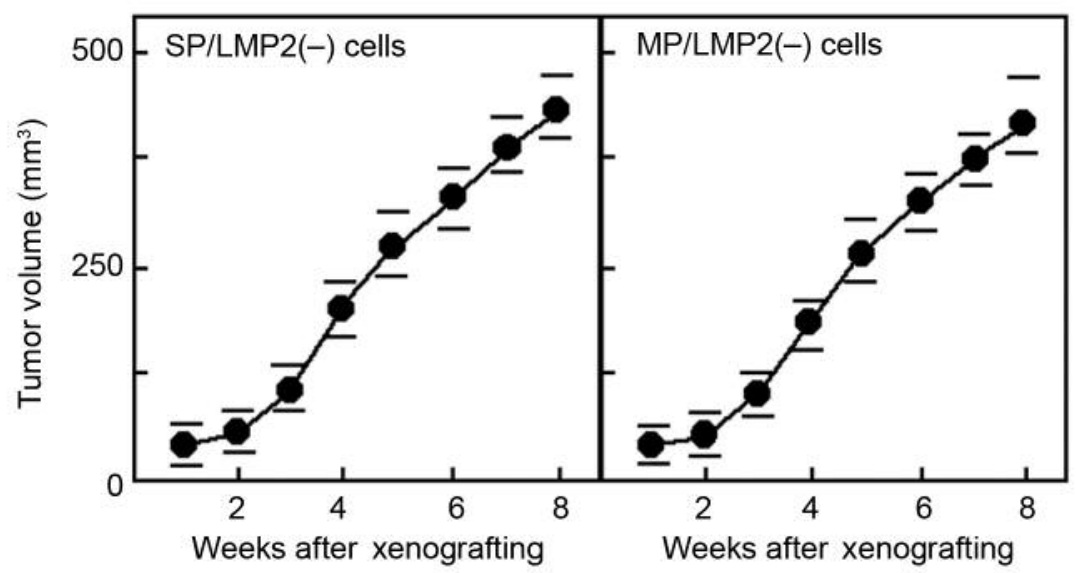

Figure 5. Xenograft studies for the micrometastasis model.SP/LMP2(-) and MP/LMP2(-) cells were harvested from a culture using trypsin, washed, and resuspended in PBS $\left(2 \times 10^{7}\right.$ cells/ml). Nude mice (BALB/cSlc-nu/nu, female, 7-8 weeks old, Japan SLC, Shizuoka, Japan) were injected with $1 \times 10^{6} \mathrm{SP} / \mathrm{LMP} 2(-)$ or MP/LMP2(-)cells in $5 \mathrm{mg} / \mathrm{ml}$ BD Matrigel Matrix (BD Biosciences) in culture medium containing $15 \%$ fetal calf serum plus SmGM-2 SingleQuots (Lonza, Basel) at a volume of $100 \mu \mathrm{l}$ on the left side of the second mammary fat pad. Tumor formation was assessed every day. Tumor volumes were calculated as $(L \times W \times W) / 2$, where $W$ represents width and $L$ represents length. Statistical analyses were performed on mean tumor volumes at the end of the study using the Student's t-test. 


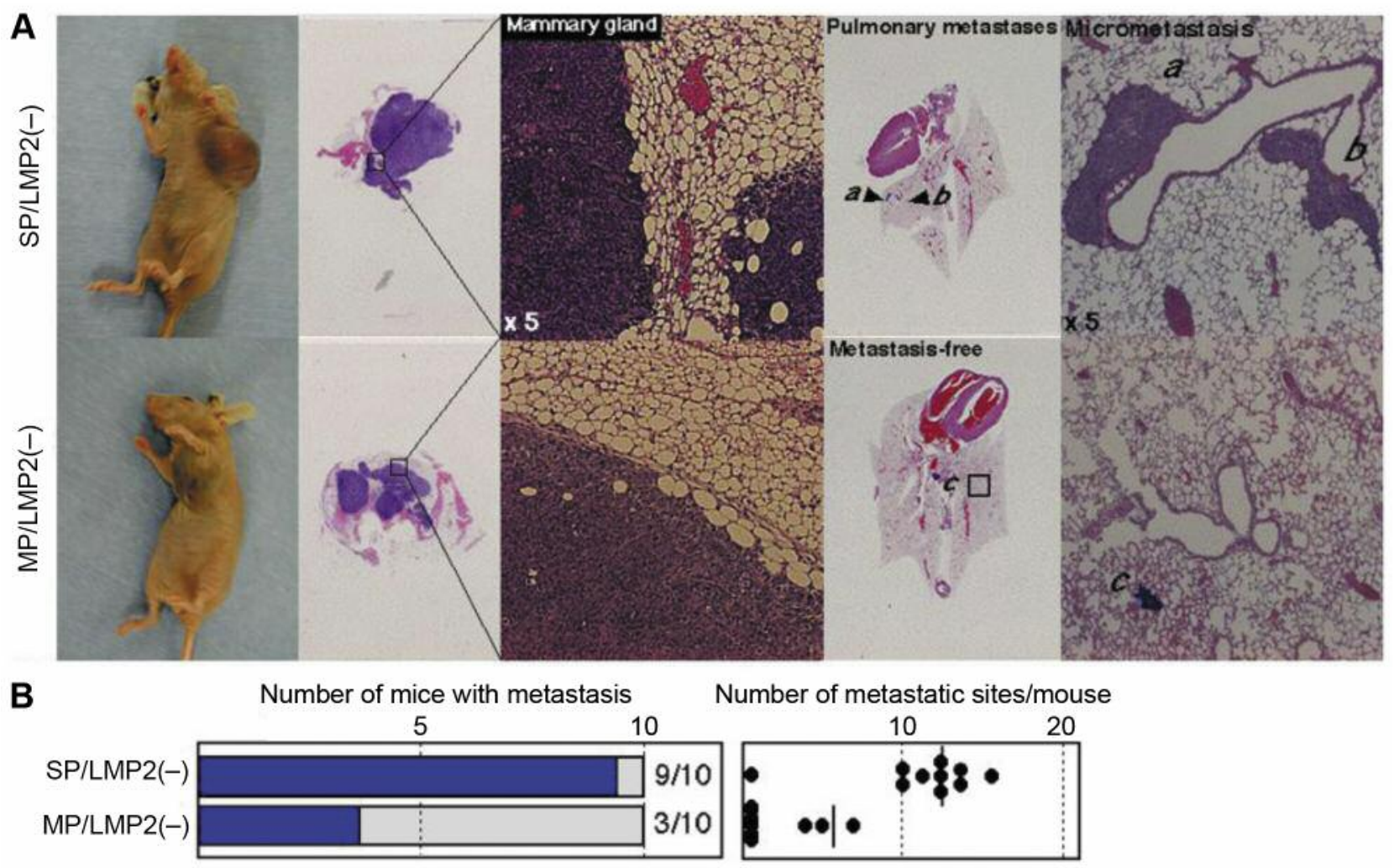

Figure 6. Induction of micrometastases in alveolar tissues derived from BALB/c nu/nu mice xenografted with human Ut-LMS stem-like cells. A. The photographs show the pathology in sections derived from the primary tumor on the left side of the second mammary fat pad and micrometastases in the alveolar tissues of BALB/c nu/nu mice xenografted with $S P / L M P 2(-)$ or MP/LMP2(-) cells 2 months after the injection. In xenograft experiments using $B A L B / c$ nu/nu mice, the same degree of tumorigenicity was observed for $S P / L M P 2(-)$ and MP/LMP2(-) xenografts. Data are representative of one out of ten independent experiments obtained with different xenograft samples derived from $S P / L M P 2(-)$ or MS/LMP2(-) cells. B. The graph on the left shows the number of mice in which micrometastases were observed in alveoli. The graph on the right shows the number of micrometastatic sites found in alveolar sections in each xenografted mouse. It is important to note that in SP/LMP2(-)-xenografted mice, the frequency of micrometastases in alveolar tissue was significantly higher than that in MP/LMP2(-)-xenografted mice. In comparison with MP/LMP2(-), Ut-LMS stem-like cells (SP/LMP2(-)) exhibit the ability to induce tumor angiogenesis and have a higher hematogenous metastatic potential.

that of VEGF-A, were significantly higher in the primary tumors of BALB/c nu/nu mice xenografted with SP/LMP2(-) cells than in those xenografted with MP/LMP2(-) cells (Figure 4). The secretion of the lymphatic endothelial growth factors VEGF-C and VEGF-D was not significantly different between primary tumors derived from SP/LMP2(-) cells and MP/LMP2(-) cells (Figure 4, Table I). Although tumorigenicity was similar for SP/LMP2(-) cells and MP/LMP2(-) cells (average tumor volumes: SP/LMP2(-) cells $409.9 \mathrm{~mm} 3, \mathrm{MP} / \mathrm{LMP} 2(-)$ cells $398.5 \mathrm{~mm}^{3}$ ), the levels of VEGF-A secreted from xenografts derived from SP/LMP2(-) cells were higher than that from xenografts derived from MP/LMP2(-) cells (average concentrations of VEGF-A: SP/LMP2(-) cells $303.6 \mathrm{pg} / \mathrm{ml}$, MP/LMP2(-) cells 913.8 $\mathrm{pg} / \mathrm{ml}$ ) (Figure 4, Table I). It is important to note that the number of micrometastases in alveolar tissues was significantly higher in BALB/c $n u / n u$ mice xenografted with $\mathrm{SP} / \mathrm{LMP} 2(-)$ cells than in BALB/c $n u / n u$ mice xenografted with MP/LMP2(-) cells (Figure 6). VEGF-A secreted from the primary tumors of $\mathrm{BALB} / \mathrm{c} n u / n u$ mice xenografted with SP/LMP2(-) cells may promote hematogenous metastasis.

To investigate the ability of human Ut-LMS stem-like cells to form endothelial vessels in vivo, we measured the relative amount of murine versus human endothelial cells within xenografts derived from $\mathrm{BALB} / \mathrm{c} n u / n u$ mice injected with SP/LMP2(-) cells or MP/LMP2(-) cells (Figure 7). The immunofluorescence staining analysis with human- and/or mouse-specific antibodies showed that UtLMS stem-like cell-derived tumors contained human vascular endothelial cells labeled with human-specific antiCD31 and human/mouse-specific anti-CD144 (VECADHERIN), whereas xenografts generated with Ut-LMS 
Potential tumour vascularization via the endothelial differentiation of uterine leiomyosarcoma stem-like cells in primary tumour

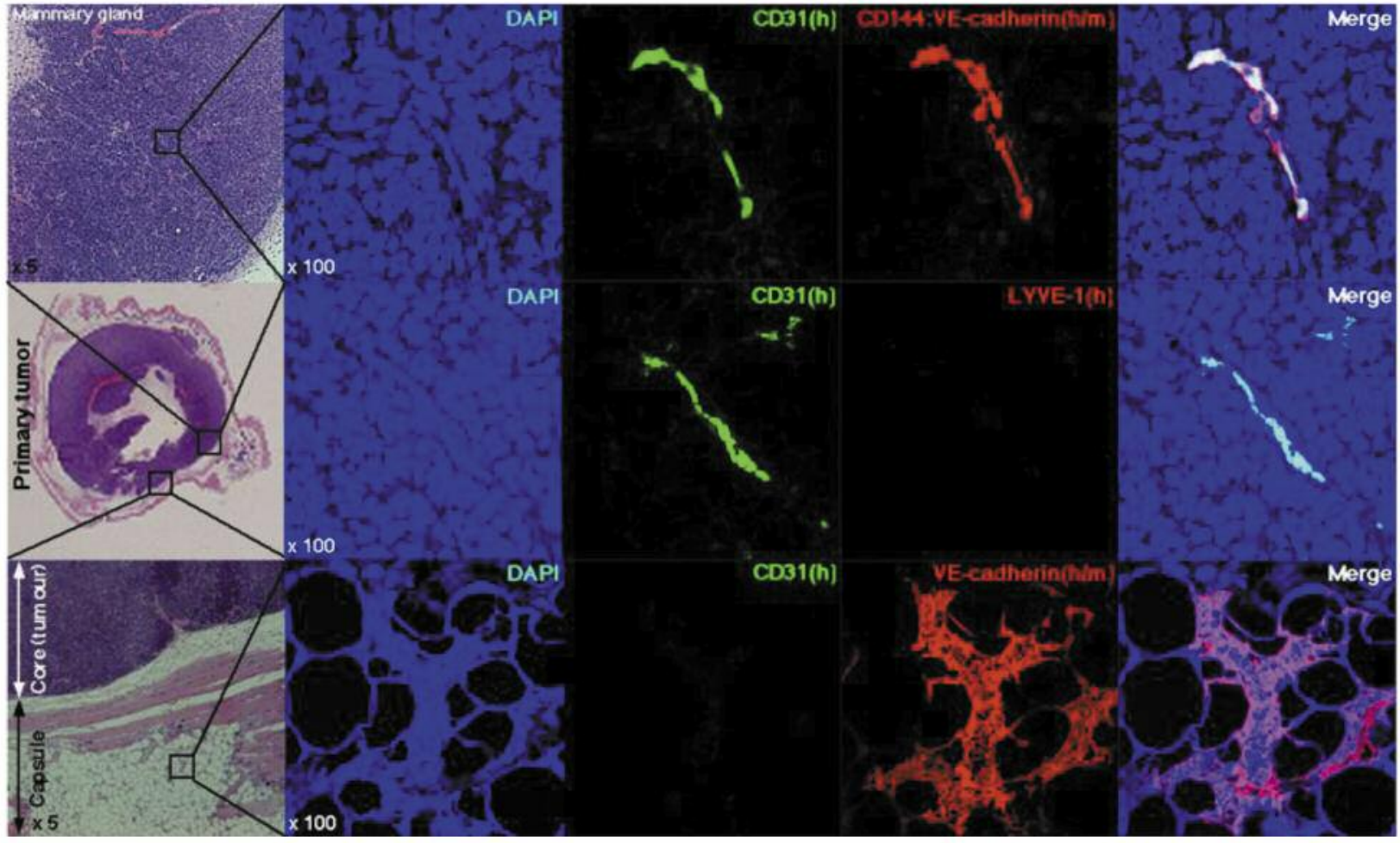

Figure 7. Human LMS stem-like cell differentiation to vascular endothelial cell progenitors. H\&E staining and immunofluorescence of tumor xenograft sections labeled with an anti-human CD31 (green), anti-human/murine CD144 (VE-CADHERIN) (red), or anti-human LYVE-1 antibody (red). Human vascular endothelial cells were detected as human CD31 and human/mouse CD144 (VE-CADHERIN) double-positive cells. Human lymphatic endothelial cells were detected as human CD31 and human LYVE-1 double-positive cells. Human LMS stem-like cells appeared to differentiate to vascular endothelial-cell progenitors, and not to lymphatic endothelial cell progenitors. Data are representative of one out of ten independent experiments obtained with different xenograft samples derived from SP/LMP2(-) or MS/LMP2(-) cells.

stem-like cells did not contain human lymphatic endothelial cells labeled with human-specific anti-CD31 and humanspecific anti-LYVE (Figure 7, Table I). Similarly, human CD31-positive cells were only detected in the core and not in the tumor capsule (Figure 3, Table 1). Endothelial vessels labeled with human/mouse-specific anti-CD144 (VE-CADHERIN) were detected in both the tumor core and capsule (Figure 7, Table I).

\section{Discussion}

The standard treatment for human Ut-LMS is resection of the uterus and bilateral appendages (oviduct and ovary). However, there is extensive evidence showing that ovariectomy does not have a marked effect on prognosis. Hematogenous metastases to the lung and the liver occur at rates $70-80 \%$ and are more important than lymphatic metastases that occur at a rate as low as 6-10\%. The reason for the higher rate of hematogenous metastasis than lymphatic metastasis remains unclear.
Cancer metastasis is mainly responsible for mortality in cancer patients and involves complex interactions between malignant and host cells that are modulated by various factors and cytokines. Vascular structures in solid tumors are crucial for cancer cell extravasation into the circulation. New blood vessel formation is important for tumor growth and anti-angiogenesis drugs have been used in cancer therapy. The discovery of VEGF-A as the primary tumor angiogenesis factor prompted the development of a number of biological agents that targeted this factor or its receptors (11, 12) (Figure 1). VEGF-B produced by tumor cells significantly remodels the tumor microvasculature, leading to leaky vascular networks that are highly permissive for tumor cell invasion. VEGF-B-induced cancer metastasis occurs through a VEGF-A-independent mechanism $(16,17)$ (Figure 1). In addition, the overexpression of VEGF-C or VEGF-D in cancer cells markedly increased intratumoral lymphangiogenesis, resulting in significantly enhanced metastasis to the regional lymph nodes and lungs $(13,14)$ (Figure 1). The degree of tumor lymphangiogenesis has been 


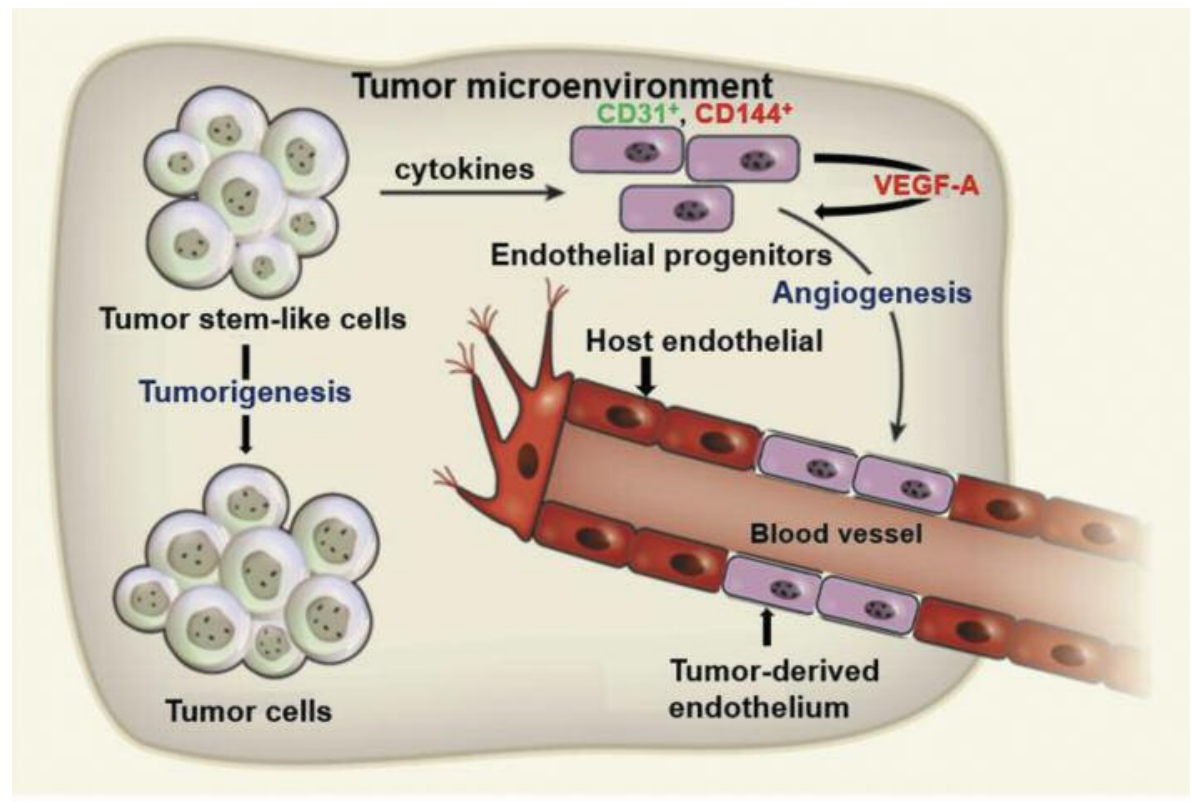

Figure 8. Transformation of cancer stem cells into vascular endothelial cells. Tumor-derived stem cells are considered to give rise to tumor cells. The present results suggest that a portion of the CD31+ and CD144+double-positive vascular endothelium that lines tumor vessels in human leiomyosarcoma also arises from tumor stem-like cells. The present study also demonstrated that the genetic abnormalities (dots) observed in tumor cells were present in endothelial cells isolated from tumors. Tumor stem-like cell differentiation to endothelial-cell progenitors appears to occur through cytokine-mediated signaling, and the further differentiation of endothelial-cell progenitors into vascular endothelial cells may be mediated by the VEGF-A signaling pathway $(22,23)$.

found to strongly correlate with the extent of lymph node and lung metastases. These findings demonstrated the occurrence and biological significance of intratumoral lymphangiogenesis in cancer and identified VEGF-C or VEGF-D as a molecular link between intratumor lymphangiogenesis and metastasis $(18,19)$ (Figure 1).

Glioblastoma cells reportedly differentiate into functional endothelial cells as part of the intratumor vasculature. These endothelial cells are characterized by the same genetic alterations as glioblastoma cells and appear to be derived from glioblastoma stem-like cells (20-23). Tumor stem-like cell differentiation to endothelial-cell progenitors appears to occur through cytokine signaling, such as Notch-mediated signaling, and the further differentiation of endothelial-cell progenitors into endothelial cells is mediated by the VEGF signaling pathway (20-23) (Figure 8). Using the OptEIA ${ }^{\text {тм }}$ set, xenografts derived from Ut-LMS stem-like cells were shown to secrete high levels of angiogenesis-inducing factor/VEGF-A, but not lymphangiogenesis-inducing factors/VEGF-C and VEGF-D. This result reflects clinical findings showing that hematogenous metastasis is frequent in human Ut-LMS. Although the angiogenic activity of mesenchymal tumor stem-like cells has not been investigated in detail in mesenchymal tumors, endothelial cells bearing tumor-specific alterations may be derived from tumor cells endowed with stem cell plasticity. Similarly, vasculogenic mimicry may represent the incomplete differentiation of Ut-LMS stem-like cells towards the endothelial lineage, as indicated by the aberrant mixed phenotype of human Ut-LMS xenografts characterized by the subset of CD31 and CD144(VE-CADHERIN) doublepositive cells, i.e. vascular endothelial cells that retain tumorigenic activity and angiogenic malignancy (24). Molecular pathological studies did not provide biological evidence to support Ut-LMS stem-like cell differentiation to CD31 and LYVE-1 double-positive cells, i.e., lymphatic endothelial cells (24) (Figure 7, Table I).

According to the present results, human Ut-LMS stem-like cells clearly exhibit a stronger ability to induce tumor angiogenesis and hematogenous metastasis than normal human Ut-LMS cells. The characteristics of these Ut-LMS stem-like cells will provide insights into the development of novel therapeutics and diagnostic methods for human UtLMS. We are now examining the physiological properties of Ut-LMS stem-like cells in more detail.

\section{Conflicts of Interest}

The Authors declare no potential conflicts of interest regarding this study. 


\section{Authors' Contributions}

T.H. and K.S. performed most of the experiments and coordinated the project; K.S. performed cell sorting and the flow cytometric analysis; T.H. detected and characterized human endothelial cells in mouse xenografts; T.H. and K.S. developed the functional assays of endothelial cell cultures; G.M. recruited patients and performed surgery; T.H., K.S., Y.K., D.Z., and H.A. were involved in pathology assessments and the detection of genomic aberrations in endothelial cells; T.H. conceived the study and wrote the manuscript. N.Y. and I.K. gave information on clinical aspects of the work and oversaw the entire study.

\section{Acknowledgements}

The Authors sincerely thank Professor Susumu Tonegawa (Picower Institute, Massachusetts Institute of Technology) for his contribution. This study was supported in part by grants from the Japan Ministry of Education, Culture, Science and Technology (No. 24592510, No. 15K1079, and No. 19K09840), the Foundation of Osaka Cancer Research, the Ichiro Kanehara Foundation of the Promotion of Medical Science and Medical Care, the Foundation for the Promotion of Cancer Research, the Kanzawa Medical Research Foundation, the Shinshu Medical Foundation, and the Takeda Foundation for Medical Science.

\section{References}

1 Wu TI, Chang T, Hsueh S, Hsu HH, Chou HH, Huang HJ and Lai $\mathrm{CH}$ : Prognostic factors and impact of adjuvant chemotherapy for uterine leiomyosarcoma. Gynecol Oncol 100: 166-172, 2006. PMID: 16182349. DOI: 10.1016/j.ygyno.2005.08.010

2 Leitao MM, Soslow RA, Nonaka D, Olshen AB, Aghajanian C, Sabbatini P, Dupont J, Hensley M, Sonoda Y, Barakat RR and Anderson S: Tissue microarray immunohistochemical expression of estrogen, progesterone, and androgen receptors in uterine leiomyomata and leiomyosarcoma. Cancer 101: 1455-1462, 2004. PMID: 15316901. DOI: $10.1002 /$ cncr.20521

3 Kurma RJ: Pathology of the Female Genital Tract, 4th ed. New York, Springer-Verlag 4, 499, 2001.

4 Diagnostic Criteria for LMS, Adapted from 2003 WHO Guidelines: World Health Organization Classification of Tumours: Pathology and Genetics, Pathology and Genetics of Tumours of the Breast and Female Genital Organs. IARC Press, France, 2003.

5 Hayashi T and Faustman D: Development of spontaneous uterine tumors in low molecular mass polypeptide- 2 knockout mice. Cancer Res 62: 24-27, 2001. PMID: 11782352.

6 Hayashi T, Kobayashi Y, Kohsaka S and Sano K: The mutation in ATP-binding region of JAK1, identified in human uterine leiomyosarcomas, results in defective interferon- $\gamma$ inducibility of TAP1 and LMP2. Oncogene 25: 4016-4026, 2006. PMID: 16474838. DOI: $10.1038 /$ sj.onc. 1209434

7 Hayashi T, Horiuchi A, Sano K, Hiraoka N, Kasai M, Ichimura T, Nagase S, Ishiko O, Kanai Y, Yaegashi N, Aburatani H, Shiozawa T and Konishi I: Potential role of LMP2 as tumorsuppressor defines new targets for uterine leiomyosarcoma therapy. Sci Rep 1: 180, 2011. PMID: 22355695. DOI: 10.1038/ sj.onc. 1209434
8 Hayashi T, Horiuchi A, Sano K, Hiraoka N, Kasai M, Ichimura T, Nagase S, Ishiko O, Kanai Y, Yaegashi N, Aburatani H, Shiozawa T, Tonegawa S and Konishi I: Potential role of LMP2 as an antioncogenic factor in human uterine leiomyosarcoma: morphological significance of calponin h1. FEBS Let 586(13): 1824-1831, 2012. PMID: 22659265. DOI: 10.1016/j.febslet.2012.05.029

9 Hayashi T, Ichimura T, Yaegashi N, Shiozawa T and Konishi I: Expression of CAVEOLIN 1 in uterine mesenchymal tumors: no relationship between malignancy and CAVEOLIN 1 expression. Biochem Biophys Res Commun 463(4): 982-987, 2015. PMID: 26072376. DOI: 10.1016/j.bbrc.2015.06.046

10 Hayashi T, Horiuchi A, Sano K, Hiraoka N, Ichimura T, Sudo T, Ishiko O, Yaegashi N, Aburatani $\mathrm{H}$ and Konishi I: Potential diagnostic biomarkers: LMP2/ $\beta 1 \mathrm{i}$ and Cyclin B1 differential expression in human uterine mesenchymal tumors. Tumori 100 : 509-516, 2014. PMID: 25296613. DOI: 10.1700/1636.17918

11 Goodell MA, Brose K, Paradis G, Conner AS and Mulligan RC: Isolation and functional properties of murine hematopoietic stem cells that are replicating in vivo. J Exp Med 183: 1797-1806, 1996. PMID: 8666936. DOI: 10.1084/jem.183.4.1797

12 Challen CA and Little MH: A side order of stem cells: the SP phenotype. Stem Cells 24: 3-12, 2006. PMID: 16449630. DOI: 10.1634/stemcells.2005-0116

13. Redvers RP, Li A and Kaur P: Side population in adult murine epidermis exhibits phenotypic and functional characteristics of keratinocyte stem cells. Proc Natl Acad Sci USA 103: 1316813173, 2006. PMID: 16920793. DOI: 10.1073/pnas.0602579103

14 Schienda J, Engleka KA, Jun S, Hansen MS, Epstein JA, Tabin CJ, Kunkel LM and Kardon G: Somitic origin of limb muscle satellite and side population cells. Proc Natl Acad Sci USA 103: 1945-950, 2006. PMID: 16418263. DOI: 10.1073/pnas.0510164103

15 Montanaro F, Liadaki K, Volinski J, Flint A and Kunkel LM: Skeletal muscle engraftment potential of adult mouse skin side population cells. Proc Natl Acad Sci USA 100: 9336-9341, 2003. PMID: 12886022. DOI: 10.1073/pnas.1133179100

16 Sitohy B, Nagy JA and Dvorak HF: Anti-VEGF/VEGFR therapy for cancer: reassessing the target. Cancer Res 72(8): 1909-1914, 2012. PMID: 22508695. DOI: 10.1158/0008-5472.CAN-11-3406

17 Yang X, Zhang Y, Hosaka K, Andersson P, Wang J, Tholander F, Cao Z, Morikawa H, Tegnér J, Yang Y, Iwamoto H, Lim S and Cao Y: VEGF-B promotes cancer metastasis through a VEGF-Aindependent mechanism and serves as a marker of poor prognosis for cancer patients. Proc Natl Acad Sci USA 112(22): E29002909, 2015. PMID: 25991856. DOI: 10.1073/pnas.1503500112

18 Skobe M, Hawighorst T, Jackson DG, Prevo R, Janes L, Velasco P, Riccardi L, Alitalo K, Claffey K and Detmar M: Induction of tumor lymphangiogenesis by VEGF-C promotes breast cancer metastasis. Nat Med 7(2): 192-198, 2001. PMID: 11175850. DOI: $10.1038 / 84643$

19 Stacker SA, Caesar C, Baldwin ME, Thornton GE, Williams PA, Prevo R, Jackson DG, Nishikawa S, Kubo H and Achen MG: VEGF-D promotes the metastatic spread of tumor cells via the lymphatics. Nat Med 7(2): 186-191, 2001. PMID: 11175849. DOI: $10.1038 / 84635$

20 Bao S, Wu Q, Sathornsumetee S, Hao Y, Li Z, Hjelmeland AB, Shi Q, McLendon RE, Bigner DD and Rich JN: Stem cell-like glioma cells promote tumor angiogenesis through vascular endothelial growth factor. Cancer Res 66(16): 7843-7848, 2006. PMID: 16912155. DOI: 10.1158/0008-5472.CAN-061010 
21 Cheng L, Huang Z, Zhou W, Wu Q, Donnola S, Liu JK, Fang X, Sloan AE, Mao Y, Lathia JD, Min W, McLendon RE, Rich JN and Bao S: Glioblastoma stem cells generate vascular pericytes to support vessel function and tumor growth. Cell 153(1): 139-152, 2013. PMID: 23540695. DOI: 10.1016/j.cell.2013.02.021

22 Ricci-Vitiani L, Pallini R, Biffoni M, Todaro M, Invernici G, Cenci T, Maira G, Parati EA, Stassi G, Larocca LM and De Maria R: Tumour vascularization via endothelial differentiation of glioblastoma stem-like cells. Nature 468(7325): 824-828, 2010. PMID: 21102434. DOI: $10.1038 /$ nature09557

23 Wang R, Chadalavada K, Wilshire J, Kowalik U, Hovinga KE, Geber A, Fligelman B, Leversha M, Brennan C and Tabar V:
Glioblastoma stem-cell like cells give rise to tumour endothelium. Nature 468(7325): 829-833, 2010. PMID: 21102433. DOI: 10.1038/nature09624

24 Baluk P and McDonald DM: Markers for microscopic imaging of lymphangiogenesis and angiogenesis. Ann NY Acad Sci 1131: 112, 2008. PMID: 18519955. DOI: 10.1196/annals.1413.001

Received December 18, 2019 Revised January 21, 2020 Accepted January 24, 2020 